\title{
Paralarvas y juveniles de Octopus bimaculatus (Cephalopoda: Octopodidae) en el Pacífico sur de México
}

\author{
Octopus bimaculatus paralarvae and juvenile (Cephalopoda: Octopodidae) \\ in the Pacific South of Mexico
}

\section{Carmen Alejo-Plata1, Rubén García-Guillén² y Jorge Herrera-Galindo ${ }^{1}$}

\begin{abstract}
${ }^{1}$ Laboratorio Ecología del Bentos, Universidad del Mar, Campus Puerto Ángel, Ciudad Universitaria, Puerto Ángel, San Pedro Pochutla, C.P: 70902, Oaxaca, México.plata@angel.umar.mx

${ }^{2}$ Licenciatura en Biología Marina, Universidad del Mar, Campus Puerto Ángel, Ciudad Universitaria, Puerto Ángel, San Pedro Pochutla, C.P. 70902, Oaxaca, México

Abstract.- The distribution and abundance of Octopus bimaculatus paralarvae and juveniles in the Pacific South of Mexico (i.e., 14 to $16^{\circ} \mathrm{N}$ ) are described. A total of 15 paralarvae were collected during three plankton surveys in July, September and November 2001. Size of paralarvae ranged from 0.7 to $2.7 \mathrm{~mm}$ Dorsal Mantle Length $(\mathrm{ML})$, with the smallest size corresponding to the $\mathrm{ML}$ at hatching. Additionally, three juveniles were collected by scuba diving in a coastal area in 2010, two small juveniles (7.4 and 9.6 mm ML) were found on a rocky area in May $\left(15^{\circ} 40^{\prime} \mathrm{N}, 96^{\circ} 29^{\prime} \mathrm{W}\right.$ ) and one big juvenile ( $30 \mathrm{~mm} \mathrm{ML}$ ) was captured in a coral reef in December $\left(15^{\circ} 44^{\prime} \mathrm{N}, 96^{\circ} 07^{\prime} \mathrm{W}\right)$. The geographic distribution of $O$. bimaculatus has been reported from California, USA to La Paz B.C.S (Mexico). This study provides new information on the abundance, distribution and size structure of early-stages of $O$. bimaculatus, redefining the geographic range of this species in the Tropical Eastern Central Pacific.
\end{abstract}

Key words: Octopus, Gulf of Tehuantepec, distribution, abundance

\section{INTRODUCCIÓN}

La familia Octopodidae contiene un gran número de especies conocidas. Algunas de ellas tienen grandes crías que son inmediatamente bentónicas (al igual que los adultos), que se denominan juveniles. Sin embargo en más de la mitad de las especies conocidas, las crías son pequeñas y planctónicas, por convención son llamadas paralarvas (Young \& Harman 1989).

Estos estadios se encuentran escasamente estudiados e inadecuadamente descritos en la mayoría de las especies (Sweeney et al. 1992); este desconocimiento se debe, en gran parte, a la relativa ineficiencia de las redes para recolectar formas juveniles, a los problemas taxonómicos para identificarlos y a la dificultad para mantener juveniles en cultivo (Vecchione 1987). Debido a esto, las paralarvas registran baja abundancia en aguas de todo el mundo y su distribución en los primeros $200 \mathrm{~m}$ de la columna de agua es un excelente indicador de la riqueza de especies en un área determinada (Haimovici et al. 2002).

En octópodos bentónicos con etapas planctónicas, las características del desove y duración de la vida planctónica parecen desempeñar un papel importante en su capacidad de dispersión (Villanueva \& Norman 2008), a veces a distancias considerables. El ciclo de vida de estas especies está relacionado con los sistemas de corrientes oceánicas, donde las puestas, las paralarvas y en algunos casos los juveniles son transportados de las áreas de desove a las zonas de alimentación y crecimiento (Trites 1983, Saito \& Kubodera 1993).

En el ámbito mundial, el pulpo es un recurso pesquero importante. En México, la pesca comercial de pulpos está basada principalmente en 5 especies del género Octopus: $O$. maya y $O$. vulgaris en el Golfo de México; $O$. macropus, O. bimaculatus y $O$. hubbsorum en el Océano Pacífico (CNP 2010) ${ }^{1}$. Además se han reportado 6 especies que no presentan importancia pesquera: O. rubescens (en Golfo de California pesca exploratoria), O. veligero, O. alecto, O. digueti, O. chierchiae y O. penicillifer (Roper et al. 1995).

Octopus bimaculatus Verrill, 1883 es un importante recurso comercial en el Golfo de California. Es una especie

${ }^{1}$ CNP. 2010. Carta Nacional Pesquera 2010. CONAPESCA, SAGARPA, Méxixo. [en línea] <http://www.conapesca.sagarpa.gob.mx/ wb/cona/actualizacion_de_la_carta_nacional_pesquera_2010> 
de la zona costera rocosa de la zona intermareal y submareal (Ambrose 1982). Se distribuye desde Isla Catalina, Carolina EUA hasta La Paz, B.C.S. México (Roper et al. 1995), sin embargo el rango geográfico de distribución de esta especie es incierto. Verril (1883) propuso que $O$. bimaculatus se distribuye desde San Diego, California hasta Panamá, e incluso más al sur; pero Berry $(1910,1911)$ revisó los registros de la Universidad de California, verificó que O. bimaculatus es muy abundante en el sur de California; para los registros australes mencionados por Verril (1883) no encontró localidades. Así, de acuerdo a los primeros registros, se entiende que $O$. bimaculatus es quizá una especie restringida a California, EUA, hasta el punto de ser llamado comúnmente pulpo de dos manchas de California. Una reducción al rango propuesto por Verril (1883), actualmente no está revisado ni justificado. Probablemente existen muchos registros incorrectos, no resueltos o cuestionables de las localidades y el ámbito de distribución de $O$. bimaculatus.

En el Pacífico mexicano el estudio de los cefalópodos se ha basado en los adultos, con excepción de algunos trabajos enfocados a paralarvas de calamares (GranadosAmores et al. 2007) y desarrollo post-embrional de Octopus maya (Moguel et al. 2010). El objetivo de este estudio fue aportar información sobre la distribución de paralarvas y juveniles de $O$. bimaculatus, redefiniendo la extensión de ámbito geográfico para esta especie en el Pacífico Sur de México.

\section{MATERIALES Y MÉTODOS}

El material biológico provinó de 3 cruceros oceanográficos realizados durante el 2001 (Fig. 1a), que cubrieron las zonas costera y oceánica. Se realizaron 2 muestreos a bordo del B/O 'El Puma', propiedad de la Universidad Nacional Autónoma de México (10-15² N, 96$\left.100^{\circ} \mathrm{O}\right)$ : ECAC-II ( 10 al 22 de julio) y ECAC-III ( 30 de agosto al 5 de septiembre). Las muestras de zooplancton se obtuvieron mediante el procedimiento propuesto por Smith \& Richardson (1979).

Se realizó un tercer muestreo a bordo del B/P 'UMAR', propiedad de la Universidad del Mar entre el 27 y 30 de noviembre (14-16 $\left.02^{\prime} \mathrm{N}, 93^{\circ} 08^{\prime}-97^{\circ} 25^{\prime} \mathrm{O}\right)$. Las muestras se obtuvieron con una red tipo CalCOFI, con un diámetro de apertura de boca de $1 \mathrm{~m}$ equipada con red de luz de malla de $300 \mu \mathrm{m}$ con flujómetro. La velocidad de arrastre fue mantenida aproximadamente a 2 nudos desde superficie $\mathrm{y}$ hasta $50 \mathrm{~m}$ de profundidad.

En cada estación de muestreo se registraron datos de temperatura $\left({ }^{\circ} \mathrm{C}\right)$ y salinidad utilizando un CTD SEABIRD. Después del arrastre el material fue fijado con formalina al $4 \%$ neutralizada con borato de sodio. La biomasa zooplanctónica se determinó mediante el método del volumen desplazado. Posteriormente, los datos de biomasa se estandarizaron a $1000 \mathrm{~m}^{3}$ de agua de mar filtrada (Smith \& Richardson 1979). Las paralarvas de Octopus se separaron en su totalidad de las muestras originales. Adicionalmente, en el 2010 se recolectaron 3 a)

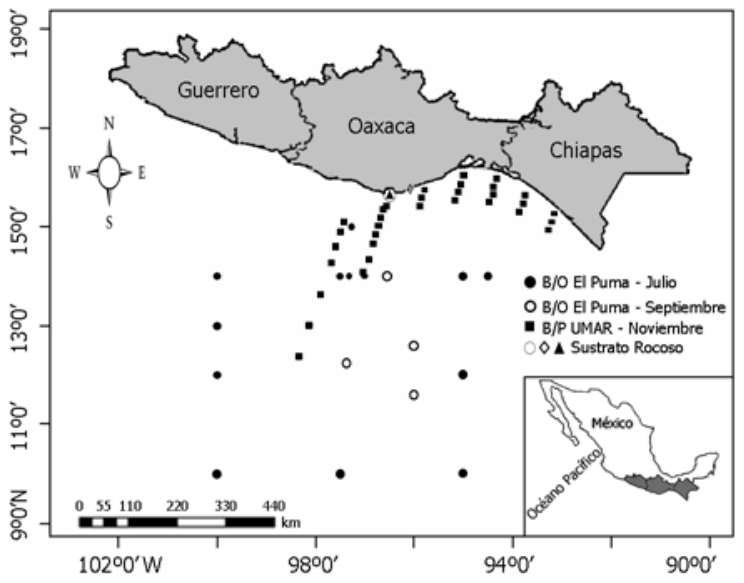

b)

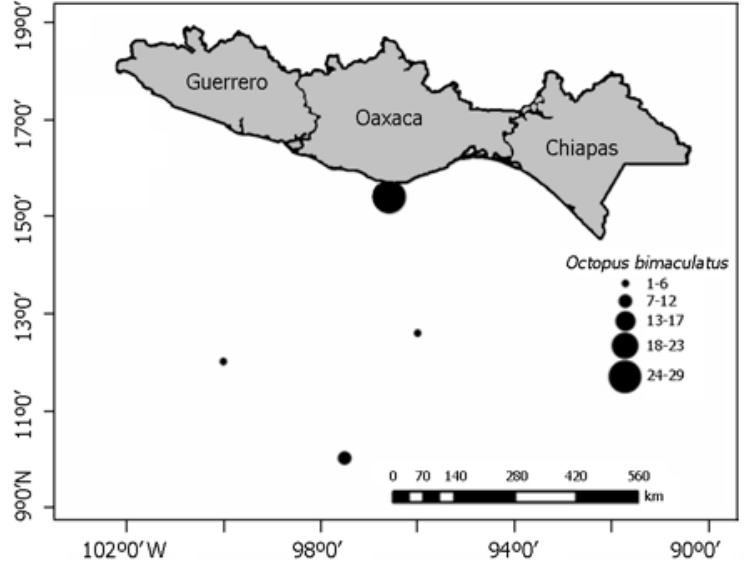

Figura 1. a) Localización de las estaciones de muestreo en el Pacífico Sur de México. Las muestras fueron tomadas durante julio, septiembre y noviembre del 2001. b) Distribución espacial y abundancia de paralarvas de Octopus bimaculatus recolectadas en el Pacífico Sur de México durante tres cruceros en 2001. Los círculos representan abundancia media por

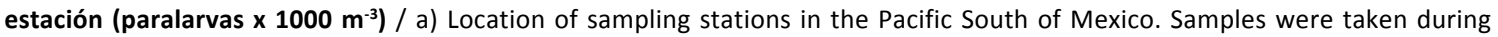
July, September and November 2001. b) Distribution and abundance of Octopus bimaculatus paralarvae in the Pacific South of Mexico in 2001. Circle area represents catch abundance (paralarvae $\times 1000 \mathrm{~m}^{-3}$ ) 
juveniles de O. bimaculatus por medio de buceo autónomo en la zona costera de Oaxaca. En mayo, a 5 m de profundidad (temperatura superficial del mar, TSM $=$ $\left.29,5^{\circ} \mathrm{C}\right)$ fueron recolectados de la zona rocosa $\left(15^{\circ} 40^{\prime} \mathrm{N}\right.$, $96^{\circ} 29^{\prime} \mathrm{O}$ ) dos juveniles pequeños, se logró mantenerlos vivas durante dos días; en diciembre se encontró un juvenil grande a $10 \mathrm{~m}$ de profundidad $\left(\mathrm{TSM}=28^{\circ} \mathrm{C}\right)$ sobre arrecife de coral $\left(15^{\circ} 44^{\prime} \mathrm{N}, 96^{\circ} 07^{\prime} \mathrm{O}\right)$.

A cada espécimen se midió la longitud dorsal del manto (LM) al 0,05 $\mathrm{mm}$ más cercano con un microscopio estereoscópico Olympus ${ }^{\circledR}$ SZX-TR30. El análisis morfológico se realizó con base en las descripciones de Sweeney et al. (1992) y Roper et al. (1995), considerando la presencia de fotóforos oculares, viscerales, así como el arreglo de cromatóforos en sifón, brazos, cabeza y manto; se utilizó una cámara lúcida para dibujar a los organismos. La especie fue confirmada por el Dr. F.G. Hochberg ${ }^{2}$.

\section{RESUlTADOS Y DISCUSIÓN}

\section{DESCRIPCIÓN DE LAS PARALARVAS}

Los especímenes analizados fueron identificados como Octopus bimaculatus y la longitud más pequeña corresponde con la esperada para la eclosión $(<3 \mathrm{~mm}$ LM) observada por Ambrose (1988) en cultivos de laboratorio. La siguiente descripción está basada en los especímenes ilustrados y siguiendo los criterios y terminología de Sweeney et al. (1992).

Los caracteres distintivos de las paralarvas de $O$. bimaculatus respecto a las otras especies de pulpo que se distribuyen en el área son el patrón de cromatóforos, el número, tamaño y distribución en el sifón, región dorsal de la cabeza, base de los brazos y manto. Los ocelos, carácter distintivo en los adultos, no son visibles en estado de paralarva y en juveniles pequeños.

Fase planctónica (Fig. 2a-f): larvas recién eclosionadas, ( $\mathrm{n}=15 ; 0,7-2,7 \mathrm{~mm} \mathrm{LM})$, brazos cortos, sub iguales con 4 ventosas; brazos con 8 cromatóforos (patrón: 1+2); sifón con 4 pequeños cromatóforos y $2+2$ cromatóforos grandes en la región media; ojos, 1 cromatóforo largo; manto, región dorsal sólo un pequeño grupo de cromatóforos en la parte posterior; región ventral cubierta uniformemente con más de 50 cromatóforos; cabeza, región dorsal con 8 cromatóforos $(2+4+2)$, región ventral,
4 cromatóforos en cada lado; cromatóforos viscerales, no visibles.

\section{FASE BENTÓNICA}

Juveniles pequeños ( $\mathrm{n}=2 ; \mathrm{LM}=7,4$ y $9,6 \mathrm{~mm}$ ), debido a que los organismos se mantuvieron vivos por 2 días se pudo observar el patrón de cromatóforos sin el efecto de los fijadores (Fig. 2g-i). Los brazos desarrollan un $2^{\circ}$ renglón de cromatóforos en el extremo distal; número y patrón de cromatóforos en sifón, cabeza y manto permanecen iguales que en la fase planctónica; cromatóforos viscerales, 1; cada hemibranquia externa con 10 laminillas; iridióforos visibles; fórmula de los brazos: $\mathrm{I}<\mathrm{IV}<\mathrm{III}<\mathrm{II}$; 36 a 38 ventosas en cada brazo. Ambrose (1988) menciona que O. bimaculatus a partir de $5 \mathrm{~mm}$ LM pasa a la fase bentónica.

Un juvenil grande, hembra inmadura, de $30 \mathrm{~mm}$ de LM (Fig. 2j), manto en forma de pera; brazos delgados con puntas atenuadas como en los adultos; fórmula de los brazos: I $<$ IV $<$ III $<$ II; brazos II y III dos veces la longitud del manto; con un ocelo muy evidente debajo de cada ojo, cerca de la base de los brazos, en el segundo y tercer par.

\section{LONGITUD DE PARALARVAS Y JUVENILES}

Las paralarvas de O. bimaculatus presentaron un intervalo de tamaño entre 0,7 y $2,7 \mathrm{~mm} \mathrm{LM}$, cercano al de los ejemplares recientemente eclosionados (Ambrose 1988), lo cual indica que la zona puede ser un área de desove para pulpos adultos y para la eclosión de sus paralarvas.

La propulsión a chorro de los pulpos durante la vida planctónica parece ser muy similar a la locomoción de los calamares, lo que sugiere que podria aumentar la capacidad de dispersión y el potencial de colonización de estas especies bentónicas. En los primeros días como paralarvas tienen un crecimiento acelerado, luego viene una disminución durante la segunda mitad de la vida planctónica. Esta interrupción en el crecimiento se produce cuando los brazos crecen y se alargan drástica y desproporcionadamente en relación con el tamaño del manto. Es en este periodo que ocurre el cambio de forma de calamar a forma de pulpo y el animal se establece en el ambiente bentónico (Villanueva et al. 1995). Para O. bimaculatus se observó que organismos $>6,0 \mathrm{~mm} \mathrm{LM}$ habitan la zona rocosa y arrecifal, siendo importante en el conocimiento del ciclo de vida de la especie.

${ }^{2}$ Department of Invertebrate Zoology, Santa Barbara Museum of Natural History, Santa Bárbara, CA 93105, USA. 

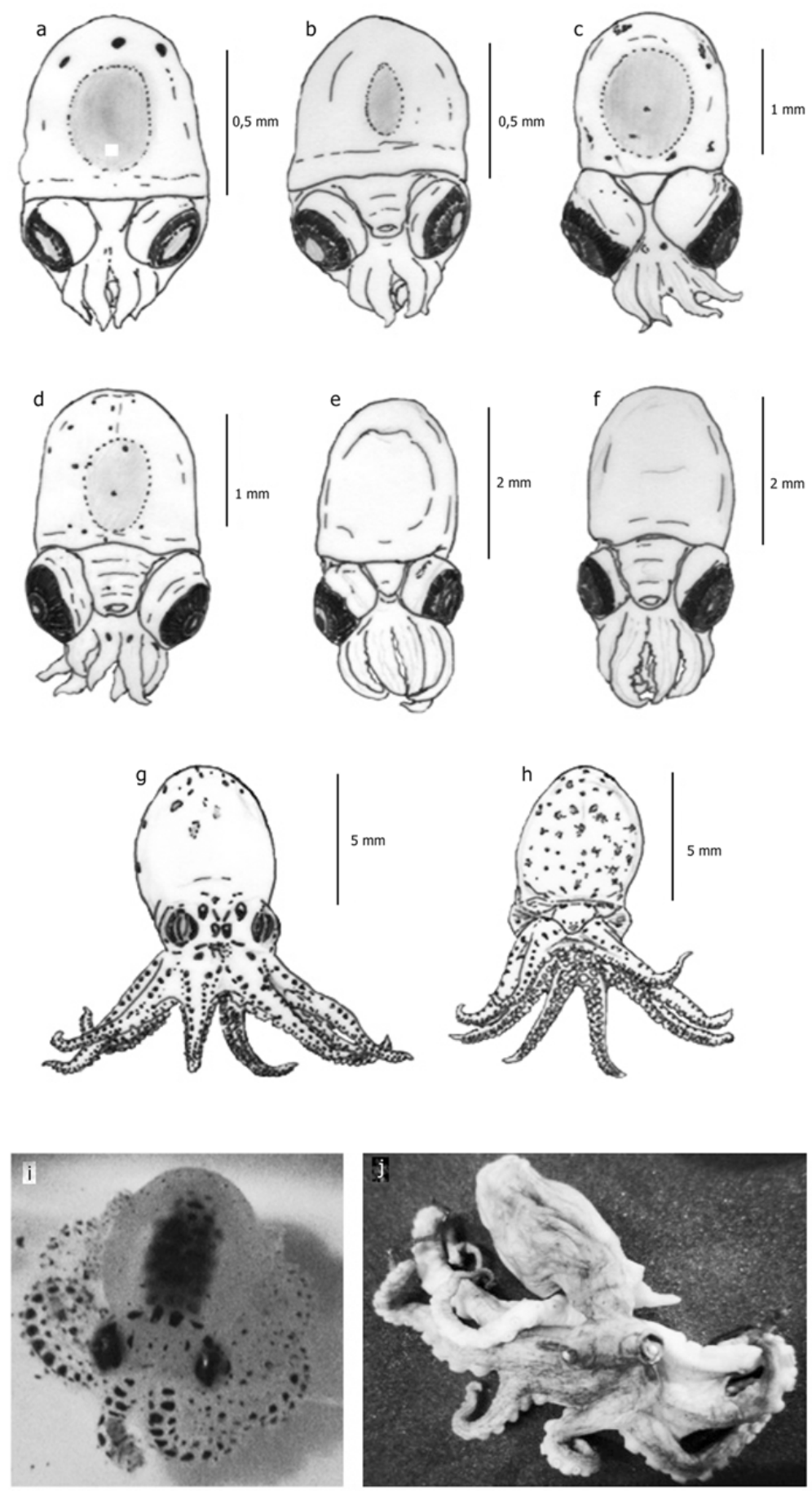

Figura 2. Octopus bimaculatus. $0,7 \mathrm{~mm} \mathrm{LM}$, a) vista dorsal, b) vista ventral; 1,2 $\mathrm{mm} \mathrm{LM,} \mathrm{c)} \mathrm{vista} \mathrm{dorsal,} \mathrm{d)} \mathrm{vista} \mathrm{ventral;} \mathrm{2,5} \mathrm{mm}$ LM, e) vista dorsal, f) vista ventral; 7,4 $\mathrm{mm} \mathrm{LM}, \mathrm{g}$ ) vista dorsal, h) vista ventral, i) vista dorsal de un espécimen vivo, j) hembra vista dorsal ( $30 \mathrm{~mm} L \mathrm{~L}$ ) con un ocelo muy evidente debajo de cada ojo, cerca de la base de los brazos, en el segundo y tercer par / Octopus bimaculatus. $0.7 \mathrm{~mm} \mathrm{ML}$, a) dorsal view, b) ventral view; $1.2 \mathrm{~mm} \mathrm{ML}, \mathrm{c}$ ) dorsal view, d) vista ventral; $2.5 \mathrm{~mm} \mathrm{ML}$, e) dorsal view, f) ventral view; $7.4 \mathrm{~mm} \mathrm{ML,} \mathrm{g)} \mathrm{dorsal} \mathrm{view,} \mathrm{h)} \mathrm{ventral}$ view, i) dorsal view living specimen, j) female dorsal view (30 $\mathrm{mm} \mathrm{ML}$ ), each conspicuous ocellum on each side of its head underneath each and near the base of the arms of the second and third pair 


\section{Abundancia y Distribución}

Se recolectaron un total de 42 muestras de zooplancton en los tres cruceros analizados. De éstas, 16 resultaron positivas para octópodos y sólo 4 estaciones lo fueron para paralarvas de $O$. bimaculatus. En total se obtuvieron 159 paralarvas de la familia Octopodidae de la cuales 15 paralarvas se determinaron como $O$. bimaculatus $(0,7$ a 2,7 mm LM).

Las paralarvas de $O$. bimaculatus fueron encontradas en toda el área de muestreo, la mayoría de ellas se concentró en las estaciones localizadas cerca de la costa, sobre la plataforma continental. Los valores de abundancia por estación variaron entre 0,7 y 29 paralarvas de $O$. bimaculatus por $1000 \mathrm{~m}^{-3}$ de agua de mar filtrada (Fig. 1b). Si bien son valores bajos, se sabe que a nivel mundial usualmente el número de paralarvas capturadas en una muestra de zooplancton varía de 1 a 5 (Vecchione 1987, Sweeney et al. 1992).

Los resultados sugieren que la presencia de las paralarvas tiene una relación estrecha con las condiciones oceanográficas del Golfo de Tehuantepec, las paralarvas pequeñas se encontraron en la estación lluviosa (TSM, $29 \geq 33^{\circ} \mathrm{C}$, salinidades de 33,1 a 34,5 ), y las más grandes $(1,2$ a $2,7 \mathrm{~mm} \mathrm{LM})$ se observaron en noviembre cuando ocurren los vientos tehuanos.

El Golfo de Tehuantepec es una región altamente productiva y de gran complejidad ecológica, debido a sus altos niveles de precipitación pluvial durante la época de lluvias (junio-octubre) y a la presencia de una surgencia eólica en la temporada de tehuanos (noviembre-abril). Durante esta última temporada, el Golfo de Tehuantepec actúa como una bomba de nutrientes, favoreciendo valores altos en la concentración de clorofila $a$ y productividad primaria (Lara-Lara et al. 1998).

Para los octópodos, el periodo entre el desarrollo embrionario y la eclosión mantiene una relación inversamente proporcional con la temperatura (Vecchione 1987). Para California, Ambrose (1988) menciona que el apareamiento de $O$. bimaculatus ocurre entre mayo y junio, la mayoría de las hembras pusieron los huevos de abril hasta agosto $\left(15-20^{\circ} \mathrm{C}\right)$ debido a que las temperaturas cálidas favorecen el desove. Por su parte, CastellanosMartínez (2008) menciona que O. bimaculatus en el Golfo de California presenta alternancia de generaciones con dos periodos de eclosión, al inicio y final de la temporada reproductiva. Así, para la zona de estudio, las paralarvas pequeñas de julio pueden corresponder a huevos eclosionados en junio, y las de noviembre a huevos eclosionados al final de la temporada reproductiva. Al respecto, Ambrose (1988) encontró que a temperaturas mayores a $30^{\circ} \mathrm{C}$ el estado paralarva de $\mathrm{O}$. bimaculatus dura 30 días, cuando las temperaturas son frías, este estadio puede durar de 2 a 3 meses.

Es evidente que se cuenta con registros incorrectos, no resueltos o cuestionables de las localidades y el ámbito geográfico de O. bimaculatus. En este trabajo se presentó una revisión exhaustiva en bases de datos de museos y colecciones de las localidades donde se ha reportado la especie a lo largo del Pacífico Tropical Oriental (CAS 2011, FMNH 2009, NMNH 2011, SIO 2011, YPM 2011). Para el Pacífico mexicano, de acuerdo a la información obtenida, las localidades de Puerto Ángel y Bahías de Huatulco en Oaxaca, deben ser incorporadas en el ámbito geográfico de la especie (Fig. 3).

La pesca artesanal de pulpo en la zona de estudio se caracteriza por efectuarse a una profundidad de $10 \mathrm{~m}$ mediante buceo autónomo, la especie que se captura es Octopus hubbsorum, y posiblemente no se ha encontrado O. bimaculatus porque se distribuye a profundidades mayores, como ocurre en el Golfo de California, donde se le ha observado hasta $10 \mathrm{~s} 50 \mathrm{~m}$ de profundidad (Castellanos-Martínez 2008). Esto se puede explicar como un falsa ausencia: lugar donde ocurre una especie y que por alguna razón no se detectó (Hirzel et al. 2002).

Muchos aspectos biogeográficos para $O$. bimaculatus son inciertos, al igual que en otras especies de octópodos del Océano Pacífico Tropical Oriental (Nesis 1987, Voight 1998). Pérez-Lozada et al. (2002) a través de evaluaciones sobre filogenia molecular encontraron que $O$. maya es una especie cercana a las especies americanas, y que es parte de una radiación adaptativa de un ancestro común. Se ubican tres grupos de pulpos: los de California, $O$. bimaculatus y $O$. bimaculoides; el de $O$. maya en el Atlántico; y el de los sudamericanos: $O$. mimus y $O$. oculifer. La separación de estas especies fue producto del surgimiento del istmo de Panamá, lo que permitió los procesos de especiación entre especies que hoy habitan el Pacífico, el Caribe y el Golfo de México. Lo anterior acentúa un origen tropical para $O$. bimaculatus y su ámbito de distribución en el Pacífico mexicano.

Los patrones de distribución de paralarvas y juveniles proporcionan evidencia adicional que Octopus bimaculatus se distribuye en el Pacífico Sur de México. Siendo éste el primer registro para la especie en el Pacífico Tropical Oriental, y redefiniendo su ámbito geográfico de distribución. 


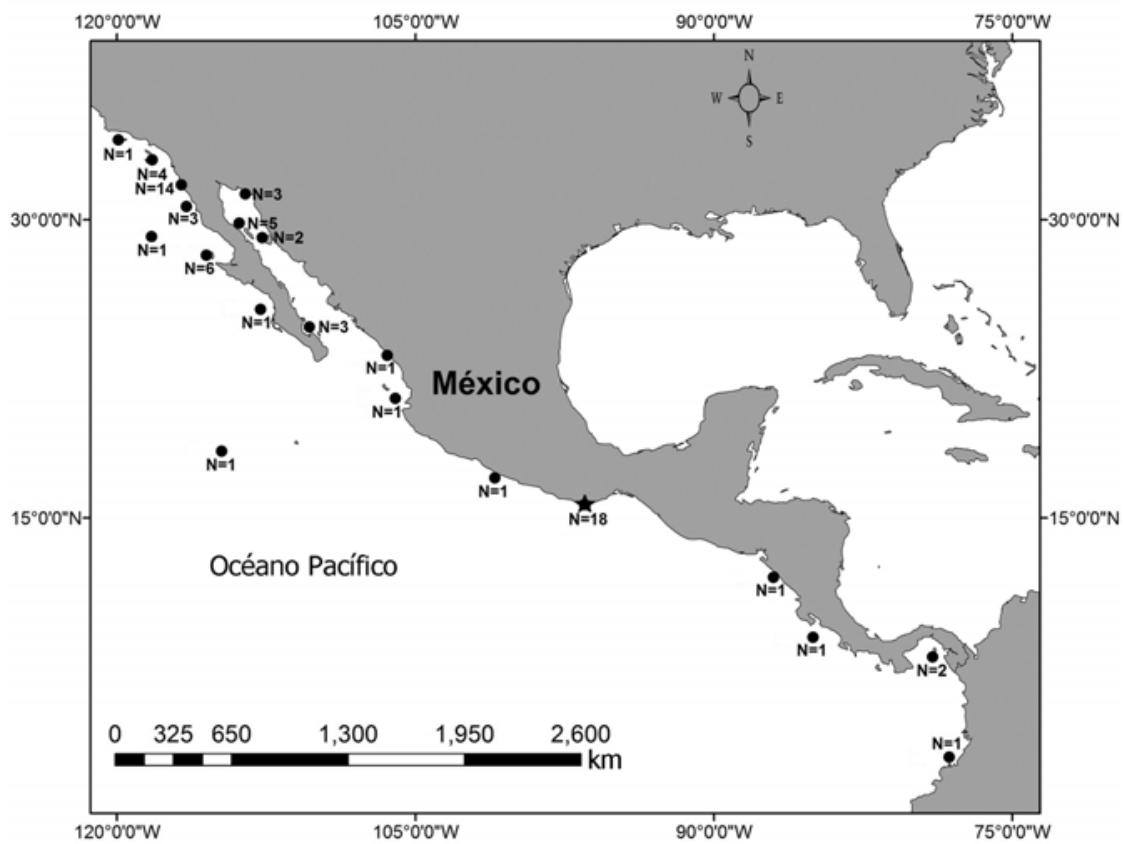

Figura 3. Localidades y ámbito de distribución de Octopus bimaculatus en el Pacífico Tropical Oriental. N, número total de registros en cada localidad / Localities and range of Octopus bimaculatus along the Eastern Tropical Pacific. N, total number of records for each locality

\section{Agradecimientos}

Los autores agradecen a Víctor Magaña Rueda del Instituto de Ciencias de la Atmósfera (UNAM) por proporcionar las muestras zooplancton cruceros ECAC; a Raúl Ramírez Barragán por los dibujos y a Sac-nicté Herrera Alejo por las fotografías. El manuscrito se enriqueció por las sugerencias de dos revisores anónimos.

\section{LITERATURA CITADA}

Ambrose RF. 1982. Shelter utilization by the molluscan cephalopod Octopus bimaculatus. Marine Ecology Progress Series 7: 67-73.

Ambrose RF. 1988. Population dynamics of Octopus bimaculatus: Influence of life history patterns, synchronous reproduction and recruitment. Malacologia 29: 23-39.

Berry SS. 1910. A review of the cephalopods of Western North America. Bulletin of the Bureau of Fisheries 30: 267-336.

Berry SS. 1911. Notes on some cephalopods in the collection of the University of California. University of California Publications in Zoology 8: 301-310.

CAS. 2011. Search the Collections Catalog. California Academy of Sciences. [on-line] $<\mathrm{http}$ ://research.calacademy.org/ redirect? url=http//researcharchive.calacaademy.org/ research/izg/iz_coll_db/index.asp>
Castellanos-Martínez S. 2008. Reproducción del pulpo Octopus bimaculatus Verril, 1983 en Bahía de los Ángeles, Baja California, México. Tesis Maestría en Ciencias, CICIMAR-IPN, La Paz, 81 pp. [en línea] $<$ http:// itzamna.bnct.ipn.mx:8080/dspace/bitstream/123456789/ 4335/1/PRODUCCPULPO.pdf>

FMNH. 2009. Invertebrate Collections Database. Field Museum of Natural History. [on-line] <http:// emuweb.fieldmuseum.org/iz/mollusks.php>

Granados-Amores J, R De Silva-Davila \& ME HernandezRivas. 2007. Epiplanktonic squids from the west coast of the Baja California Peninsula, Mexico. En: Olson RJ \& JW Young (eds). The role of squid in open ocean ecosystems. Report of GLOBEC-CLIOTOP/PFRP Workshop, pp. 1617 November 2006, Honolulu, Hawaii, USA. GLOBEC Report 24: 22-24.

Haimovici M, U Piatkowski \& R Aguiar dos Santos. 2002. Cephalopod paralarvae around tropical seamounts and oceanic island do of the northeastern coast of Brazil. Bulletin of Marine Science 71: 313-330.

Hirzel A, J Hausser, D Chessel \& N Perrin. 2002. Ecologicalniche factor analysis: how to compute habitat-suitability maps without absence data? Ecology 83(7): 2027-2036. 
Lara-Lara JR, EG Robles-Jerero, MC Bazán-Guzmán \& E Millán-Nuñez. 1998. Productividad del fitoplancton. En: Tapia-Garcia M (ed). El Golfo de Tehuantepec: el ecosistema y sus recursos, pp. 51-58. Universidad Autónoma Metropolitana-Iztapalapa, México.

Moguel C, M Mascaro, OH Avila-Poveda, C CaamalMonsreal, A Sanchez, C Pascual \& C Rosas. 2010. Morphological, physiological and behavioral changes during post-hatching development of Octopus maya (Mollusca: Cephalopoda) with special focus on the digestive system. Aquatic Biology 9: 35-48.

Nesis KN. 1987. Cephalopods of the world: squids, cuttlefishes, octopus and aliens, 334 pp. T.F.H. Publications, Neptune City.

NMNH. 2011. Invertebrate Zoology Collections Search. Smithsonian National Museum of Natural History. [online] <http://collections.nmnh.si.edu/search/iz $>$

Pérez-Lozada M, A Guerra \& A Sanjuan. 2002. Allozyme divergence supporting the taxonomic separation of Octopus mimus and Octopus maya from Octopus vulgaris (Cephalopoda: Octopoda). Bulletin of Marine Science 71: 653-664.

Roper CFE, MJ Sweeney \& FG Hochberg. 1995. Cefalópodos. En: Fisher W, F Krupp, C Sommer, KE Carpenter \& VH Niem (eds). Guía FAO para la identificación de especies para los fines de pesca Pacifico Centro-Oriental. Vol. I. Plantas e invertebrados, pp. 235253. FAO, Rome.

Saito H \& T Kubodera. 1993. Distribution of Ommastrephid Rhynchoteuthion paralarvae (Mollusca, Cephalopoda) in the Kuroshio Region. En: Okutani T, RK O'Dor \& T Kubodera (eds). Recent advances in fisheries biology, pp. 457-466. Tokai University Press, Tokyo.
SIO. 2011. Bi Collection. Scripps Institution of Oceanography [on line] $<$ http/collections.ucsd.edu/bi/search/>

Smith PE \& SL Richardson. 1979. Técnicas modelo para prospección de huevos y larvas de peces pelágicos. FAO Documento Técnico de Pesca 175: 1-107.

Sweeney MJ, CFE Roper, KM Mangold, MR Clarke \& S Boletzky. 1992. 'Larval' and juvenile cephalopods: A manual for their identification. Smithsonian Contribution to Zoology 513: 1-282.

Trites RW. 1983. Physical oceanographic features and processes relevant to Illex illecebrosus spawning in the western North Atlantic and subsequent larval distribution. NAFO Science Council Study 6: 39-55.

Vecchione M. 1987. Juvenile ecology. En: Boyle PR (ed). Cephalopod life cycles, Volumen II, Comparative reviews, pp. 61-84. Academic Press, London.

Verril AE. 1883. Descriptions of two species of Octopus from California. Bulletin of the Museum of Comparative Zoology at Harvard College, Cambridge 11: 117-124.

Villanueva R \& MD Norman. 2008. Biology of the planktonic stages of benthic octopuses. Oceanography and Marine Biology: An Annual Review 46: 105-202.

Villanueva R, C Nozais \& S Boletzky. 1995. The planktonic life of Octopus. Nature 377: 107.

Voight J. 1998. An overview of shallow water Octopus biogeography. Smithsonian Contribution to Zoology 586: 549-559.

YPM. 2011. Yale Peabody Museum-Catalog Service. Yale Peabody Museum [on-line] <http://peabody.yale.edu/ collections/search-collections?iz $>$

Young RE \& RF Harman. 1989. 'Larva', 'paralarva' and 'subadult' in cephalopod terminology. Malacologia 29(1): 201-207.

Recibido el 24 de noviembre de 2011 y aceptado el 7 de junio de 2012

Editor Asociado: Mauricio Landaeta D. 